Preprint to be published in Laser-Induced Damage in Optical Materials: 2006

SPIE Vol. 6403

\title{
XTREME OPTICS - THE BEHAVIOR OF CAVITY OPTICS FOR THE JEFFERSON LAB FREE-ELECTRON LASER
}

\author{
Michelle Shinn*a $^{*^{\mathrm{a}}}$, Christopher Behre ${ }^{\mathrm{b}}$, Stephen Benson ${ }^{\mathrm{a}}$, David Douglas ${ }^{\mathrm{a}}$, Fred Dylla ${ }^{\mathrm{a}}$, Christopher \\ Gould $^{\mathrm{a}}$, Joseph Gubeli ${ }^{\mathrm{a}}$, David Hardy ${ }^{\mathrm{a}}$, Kevin Jordan ${ }^{\mathrm{a}}$, George Neil ${ }^{\mathrm{a}}$, and Shukui Zhang ${ }^{\mathrm{a}}$ \\ ${ }^{a}$ Thomas Jefferson National Accelerator Facility, 12000 Jefferson Ave, Newport News, VA, USA \\ 23606 \\ b now at Naval Surface Warfare Center, Dahlgren Division, Code Q22, Dahlgren, VA USA 22448
}

\begin{abstract}
The cavity optics within high power free-electron lasers based on energy-recovering accelerators are subjected to extreme conditions associated with illumination from a broad spectrum of radiation, often at high irradiances. This is especially true for the output coupler, where absorption of radiation by both the mirror substrate and coating places significant design restrictions to properly manage heat load and prevent mirror distortion. Besides the fundamental lasing wavelength, the mirrors are irradiated with light at harmonics of the fundamental, $\mathrm{THz}$ radiation generated by the bending magnets downstream of the wiggler, and x-rays produced when the electron beam strikes accelerator diagnostic components (e.g., wire scanners and view screens) or from inadvertent beam loss. The optics must reside within high vacuum at $\sim 10^{-8}$ Torr and this requirement introduces its own set of complications. This talk discusses the performance of numerous high reflector and output coupler optics assemblies and provides a detailed list of lessons learned gleaned from years of experience operating the Upgrade IR FEL, a $10 \mathrm{~kW}$-class, sub-ps laser with output wavelength from 1 to 6 microns.
\end{abstract}

Keywords: Free-electron lasers, high average power lasers, ultrafast lasers, resonators

\section{INTRODUCTION}

The Free-electron laser (FEL) User Facility at Jefferson Lab (JLab) saw first light in 1998, and ran as a User Facility from 1999-2001. We then decommissioned our first FEL, the IR Demo, so we could install the IR Upgrade, which had almost an order of magnitude higher output and a larger tuning range. We once again began providing light to users in 2005. Some of the unique features of this FEL, the User facility, and applications are contained in past proceedings of this and other conferences ${ }^{1-3}$

When operating the FEL for users, one pays more attention to laser properties like output power and beam position stability than if beam delivery were into a dump a meter or two away. Commissioning activities become more than achieving a specified parameter for seconds or minutes, we need to maintain them simultaneously for hours. While operating the IR Demo, we saw the need to improve our handling of scattered and absorbed laser radiation in both cavity and beam transport components, and so we took the opportunity to incorporate these improvements in the Upgrade. This paper gives details about some of these improvements and of equal importance, points out where additional mitigation efforts are required.

*shinn@jlab.org; phone 1757 269-7565; fax 1757 269-6384 www.jlab.org/fel 
Preprint to be published in Laser-Induced Damage in Optical Materials: 2006

SPIE Vol. 6403

\section{DESIGN CONSIDERATIONS}

\section{The optical cavity environment}

The specifications for the IR Upgrade optical cavity were more or less set in $2002^{4}$ and are reproduced in Table 1 .

Table 1 Specifications for the IR Upgrade optical cavity

\begin{tabular}{ll}
\hline Quantity & Value \\
\hline Environment & Vacuum; 1 x $10^{-8}$ Torr or better \\
Cavity length & $32.042 \mathrm{~m}$ \\
Translational repeatability* & $0.10 \mathrm{~mm}$ \\
Angular repeatability* & $0.2 \mathrm{mrad}$ \\
Angular stability (passive) & $1 \mu \mathrm{rad}$ \\
Angular stability (active) & $0.2 \mu \mathrm{rad}(1 \mathrm{~Hz}-100 \mathrm{~Hz})$ \\
Length stability (passive) & $<1 \mu \mathrm{m} / \mathrm{day}$ \\
Circulating Intensity & $100 \mathrm{~kW} / \mathrm{cm}^{2}$ incident, $\leq 100 \mathrm{ppm}$ absorbed \\
Aberration (at laser wavelength) & $<\mathrm{l} / 20 \lambda \mathrm{P}-\mathrm{V}$ surface distortion \\
& $<\mathrm{l} / 5 \lambda \mathrm{P}-\mathrm{V}$ transmitted wavefront distortion. \\
\hline
\end{tabular}

* after mirror change

A schematic representation of the JLab FEL is shown in Fig. 1.

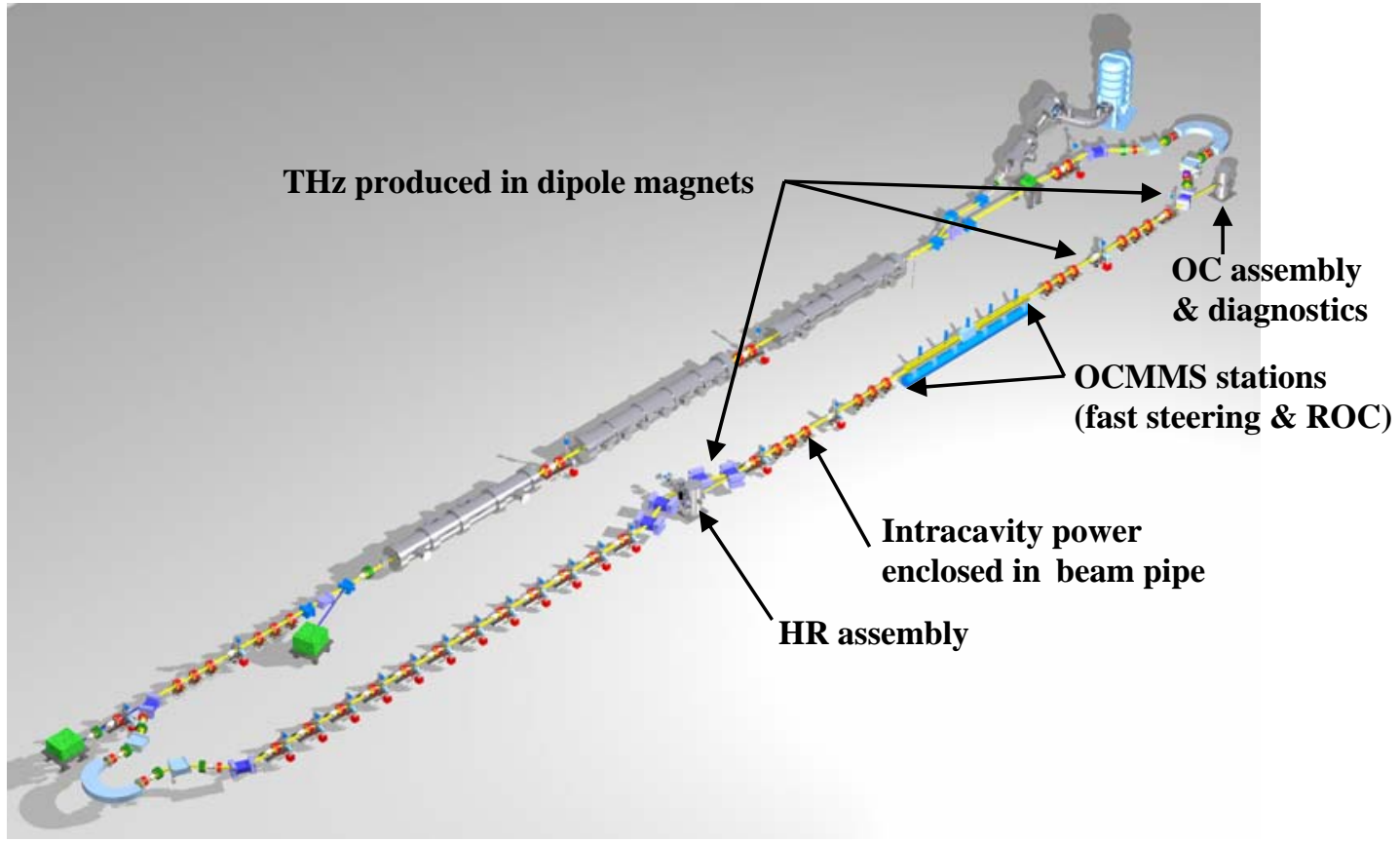

Fig. 1 The IR Upgrade FEL, with an emphasis on the optical cavity subsystems and sources of THz radiation.

We fairly easily achieved the mechanical specs, but the optical specs were more elusive. The absorptive losses were higher than spec, initially by over an order of magnitude, and aberrations that were induced by our mirror mounting practices were about 4 times higher than spec. Absorptive loss values were improved by switching vendors, and the aberrations were reduced by improving our mounting techniques, though we often find that the optical figure is worse 
when installed in the cavity, relative to when it was mounted in the same way in our test stand. This is being addressed and will be documented elsewhere.

With the exception of the vacuum and irradiance specs, the environment the cavity optics experience doesn't appear to be too extreme. However, one must also consider that the high average power output (multi-kW) comes in the form of a pulsetrain with repetition rates in the many 10's of $\mathrm{MHz}$ with each pulse having a sub-ps pulselength. Consider these typical operating conditions: the optical mode at the mirrors is a Gaussian with a $1 / \mathrm{e}^{2}$ radius of $10 \mathrm{~mm}$, the outcoupling is $10 \%$, the output power is $3 \mathrm{~kW}$, and the pulse repetition frequency (PRF) is $18.71 \mathrm{MHz}$. The average power irradiance at the center of the mode is $\sim 19 \mathrm{~kW} / \mathrm{cm}^{2}$ and the peak power irradiance (from each pulse) is in excess of $5 \mathrm{GW} / \mathrm{cm}^{2}$ ! Furthermore, the mirrors, especially the mirror downstream of the wiggler (usually the outcoupler), is irradiated with a variety of out-of-band radiation. For example, when electron beam diagnostic viewers are inserted, there are high energy (up to $\sim 100 \mathrm{MeV}$ ) bursts of $\mathrm{x}$-rays produced, with dose rates of 100s of R/hr. However, the duty factor is low. During lasing, the amount of high energy radiation lessens but is still at the level of several $100 \mathrm{mR} / \mathrm{hr}$. Besides $\mathrm{x}$-rays there is far-IR radiation, in the THz region of the spectrum ${ }^{5}$, and higher harmonics, also coherent, of the fundamental lasing wavelength, extending into the DUV region ${ }^{6}$. Total power launched from this out-of-band radiation is on the order of many 10's of watts. Finally, the sapphire outcoupler mirrors may be cooled to $\sim 50^{\circ} \mathrm{K}$ to improve their thermomechanical properties. It is the wide spectrum of radiation the cavity optics are subjected to, as well as the ambient pressure and temperature that in some ways resembles that of outer space that makes the environment extreme.

Another source of loss, often of little consequence to designers of lower-powered lasers, is scattered light. For the FEL, with it's high intracavity power, even scatter levels of order $10^{-4}$ result in several watts of scattered fundamental laser light. $\mathrm{THz}$ and second harmonic radiation considerably raise this amount, which have a large mode extent. Any scattered radiation that finds its way back into the connecting beam line (see Fig. 1) is constrained, and to some degree concentrated by diffraction and reflection, until it is absorbed somewhere. The result of this absorption manifests itself with higher pressure levels in the beam line, due to thermal desorption, often to the point that the automated machine protection system closes vacuum valves and shuts the machine off. Or, it can heat components to the point of failure, as is shown in Fig. 2, where gold-coated stainless steel screen before fused silica viewports was melted by the absorbed power.

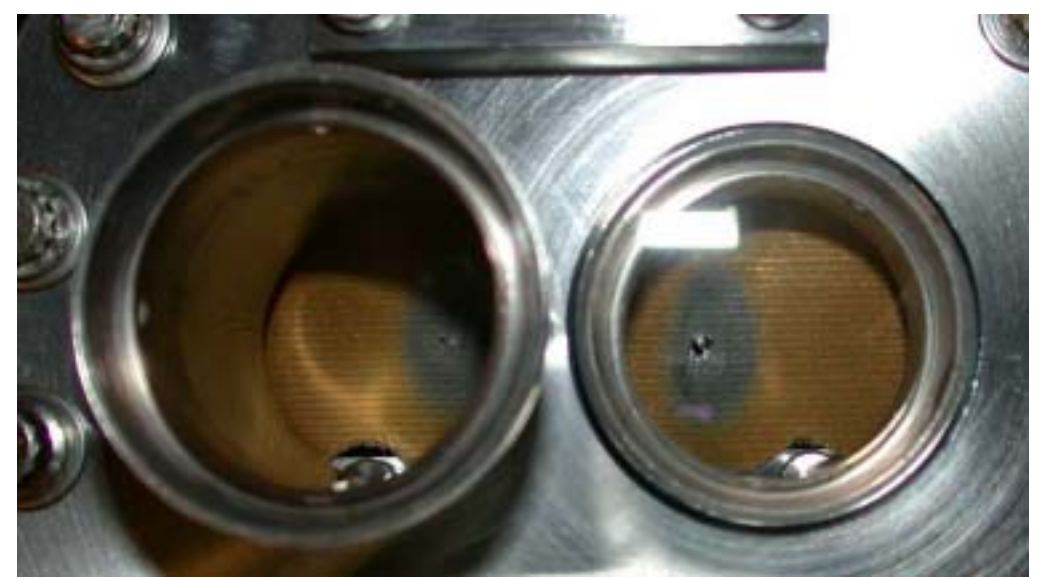

Fig. 2 The result of absorbed scattered light on gold-coated stainless steel screens placed before fused silica viewports near the wiggler.

In addition, scattered light strikes the mirror mounts, which aren't cooled and are poorly heat sunk. The mounts then warm up, outgassing and flexing. This flexing cause mode steering and forces the laser operator to make frequent adjustments to keep the power optimized.

When we were in the optical cavity design phase we considered ways to best mitigate the effects of scattered light. We used active cooling of components, as well as built cavity components of low-expansion stainless steel, specifically Invar-36. Many components were gold-coated to reflect scattered light, rather than absorb it. However, we needed to do more, as discussed in the following section. 


\section{MITIGATION STRATEGIES}

\subsection{Coherent Harmonics and THz}

From an optical cavity standpoint, $\mathrm{THz}$ mitigation takes the form of substrate selection, since the optical coatings are so thin compared to the wavelength that they don't interact. Since the $\mathrm{THz}$ radiation is launched along the electron beam direction, we are primarily concerned with that mirror, which is usually the transmissive outcoupler. If one consults the literature, diamond is the best material, but is prohibitively expensive to buy and fabricate. The next best material is sapphire, which can be fabricated by a number of vendors and is far more reasonable to procure. Our high reflectors use silicon substrates, which can be a good transmitter of THz, however our radius of curvature control mechanism, which is attached to the mirror, will absorb it, so we have an appropriately thick layer of metal (gold or aluminum, depending on the fundamental lasing wavelength) to reflect $\mathrm{THz}$ away from the substrate.

Our coating design has evolved to handle the coherent harmonics. Since they too are launched along the electron beam path, our coatings are no longer quarter-wave dielectric stacks, but are a slightly more complicated, proprietary design dubbed, "harmonic control coatings". These designs optimize the transmission of the even harmonics through the outcoupler, and reflects the odd harmonics about as well as the fundamental. These reflected odd harmonics then propagate back to the high reflector, which is also optimized to provide higher than normal reflectivity at those wavelengths. The net result was to reduce the overall absorption in the outcoupler by about $100 \mathrm{ppm}$. One is penalized in tunability, as the HR coating doesn't have the bandwidth it has with the quarter wave design. As a consequence, when you approach either end of the narrower tuning curve, many odd harmonics, which are in the visible or UV regions of the spectrum, are efficiently absorbed in the silicon.

\subsection{Scattered light}

As discussed earlier, our observations of mirror drift at the ends of the cavity, and pressure rise and melted components near the center of the cavity needed to be addressed. We devised schemes to address each region. For the mirrors, we installed water-cooled copper plates, which we call absorber shields, just in front of the mirrors. These plates are blackened to absorb most of the light incident on them, rather than allow it to heat the mirror mounts, and reflect back down the beam line. There are extensions to the shields that extend another $20 \mathrm{~cm}$ to one side. This shields the controls wiring from intercepting stray light. After some tests of different blackening processes, we chose to have the shields blackened by a commercial process known as Electroblack ${ }^{7}$. The measured reflectance at 10.6 microns was $0.3 \%$ and was anticipated to be equally low at shorter wavelengths. However, at $\mathrm{THz}$ wavelengths it may be a poor absorber, given that the surface is thinner than the wavelength. We plan to measure this in the near future. The blackened copper is very low outgassing, once the initially adsorbed water is removed. The pressure in the HR assembly, which hasn't been opened to atmosphere in over a year, is $\sim 8 \times 10^{-9}$ Torr.

Installation of the shields resulted in a marked reduction in the pressure rise associated with high average power lasing, and some reduction in mirror steering. Using thermal imaging cameras to observe the mirrors and shields, we determined that the initial design, which preserved as much clear aperture of the mirror as possible, was still allowing too much scattered light to strike the mirror mounting hardware. We installed adapter rings that reduced the diameter by $\sim 15 \%$, and steering ceased on the mirrors with the improved shields. Figure 3 is a picture of the mirrors in one of the assemblies with the current version of the shields.

Addressing the stray light problem for the Optical Cavity Mirror Metrology (OCMMS) hardware near cavity center (Fig 1), required a different solution. In this region we needed to gracefully keep the stray light from striking the viewports needed for the diagnostic beams, yet allow the beams to propagate in the same region the stray light would occupy. Our solution was to machine our own mirrors out of gold-coated Invar. These mirrors deflected the wide spectrum of radiation into a beam dump. Small holes in the mirror, whose area was only a small fraction of the total area, allow the diagnostic beams to pass. 
Preprint to be published in Laser-Induced Damage in Optical Materials: 2006

SPIE Vol. 6403

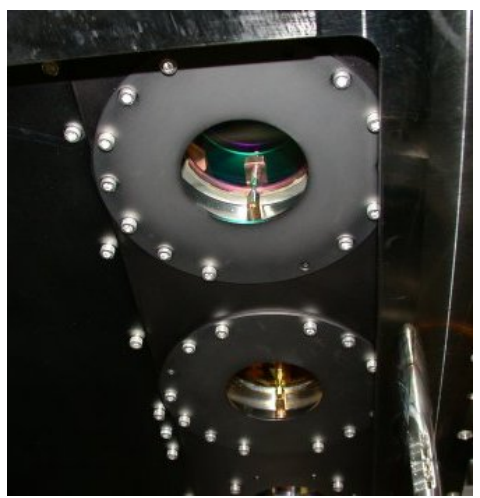

Fig. 3 Photograph of several of the mirrors in the outcoupler assembly, showing the absorber shields and the adapter rings

\section{CONCLUSIONS}

The cavity optics in a high average power FEL are in an extreme environment. They are subjected to high peak and average irradiance, out-of-band radiation at the watts to tens-of-watt level over a spectral range spanning the THz to UV range, while residing in a high vacuum. In this paper we have attempted to highlight some of our approaches to eliminating sources of loss that would degrade the operation of the laser for our users. In this environment, these losses, primarily from absorption of scattered light, and out-of-band radiation from THz and coherent harmonics, required novel solutions. We intend to use them for proposed upgrades to the current FEL, as well as for the UV-FEL. As some of our approaches are still being evaluated, they may well evolve as we collect more performance data.

\section{ACKNOWLEDGEMENTS}

The authors would like to thank their coworkers in the FEL Division as well as staff at Jefferson Lab for their assistance in building and operating this unique facility. In particular, the first author would like to express her appreciation to Gwyn Williams for teaching her much about the creation of THz radiation with short bunch length electrons. Our work was supported by U.S. DOE Contract No. DE-AC05-84-ER40150, the Office of Naval Research, the Air Force Research Laboratory, the Army Night Vision Laboratory, the Commonwealth of Virginia and the Laser Processing Consortium.

\section{REFERENCES}

1. M. D. Shinn, et al., "The Jefferson Lab FEL User Facility,” in Laser-Induced Damage in Optical Materials: 1999, Gregory Exharos, Arthur Guenther, Keith Lewis, M. J. Soileau, Editors, Proceedings of SPIE 3902, pp. 355-360, (2000).

2. M. D. Shinn, "High Average Power Free-Electron Lasers - A New Laser Source for Materials Processing" SPIE 4065, pp 434-440(2000).

3. S.V. Benson, "High Power Lasing in the IR Upgrade FEL at Jefferson Lab", Proc. 26th Int. FEL Conf. (Trieste) http://accelconf.web.cern.ch/AccelConf/f04/papers/TUCOS02/TUCOS02.PDF

4. M.D. Shinn, G. R. Baker, C. P. Behre, S. V. Benson, M. E. Bevins, L.A. Dillon-Townes, H. F. Dylla, E. J. Feldl, J. F. Gubeli, R. D. Lassiter, F. D. Martin, and G. R. Neil "Design of the Jefferson Lab IR Upgrade FEL Optical Cavity,” Nuclear Instruments and Methods A507, pp 196-199 (2003).

5. G.L. Carr, M.C. Martin, W.R. McKinney, K. Jordan, G.R. Neil, and G.P. Williams, "High Power Terahertz Radiation from Relativistic Electrons”, Nature 420 pp 153-156 (2002

6. S.V. Benson, J.F. Gubeli III, and M.D. Shinn, "Coherent Harmonics in the Super-Radiant Regime from an FEL", Proc. of the 2001 Particle Accelerator Conference, available at http://epaper.kek.jp/p01/PAPERS/WPPH117.PDF

7. Electroblack is a proprietary process developed by the Enequist Chemical Co, Inc, Glen Cove, NY (800) 521-5520 